REVISTA ANDALUZA DE ANTROPOLOGÍA.

NÚMERO 13:

OCTUBRE DE 2017

ISSN 2174-6796

[pp. 1-24]

http://dx.doi.org/10.12795/RAA.2017.13.01

Fecha de Recepción: 01/05/2017

Fecha de Aceptación: 23/06/2017

\title{
APUNTES PARA UNA HISTORIA COMUNICATIVA DE LOS KICHWA-SARAGURO: DE LA KIPA AL TELÉFONO MÓVIL
}

\section{NOTES FOR A COMMUNICATIVE HISTORY OF THE KICHWA-SARAGURO: FROM KIPA TO MOBILE PHONE}

\author{
Saleta de Salvador Agra \\ Universidade de Vigo
}

Yolanda Martínez Suárez

Universitat Autònoma de Barcelona (InCom-UAB)

\section{Resumen.}

Se ha escrito mucho sobre los indígenas Saraguro, sin embargo, la aproximación tecnológica a estas comunidades kichwa, residentes en la zona interandina y amazónica ecuatorianas, es prácticamente inexistente. En este artículo, centrándonos en el teléfono móvil, proponemos una fotografía fija de los actuales usos comunicativos de las comunidades Saraguro de la Amazonía ecuatoriana. Hemos seleccionado el teléfono móvil porque su introducción en dichas comunidades produjo cambios no sólo a nivel extracomunitario sino también intracomunitario, desplazando a la tradicional kipa como forma de comunicación. Los teléfonos móviles asumen esos y otros usos tradicionales de comunicación Saraguro, y son un ejemplo paradigmático de apropiación mediática. El estudio en el que se fundamenta empíricamente este artículo se desarrolló en la provincia 
sur-oriental de Ecuador, Zamora Chinchipe, entre marzo y noviembre de 2014. La información se recogió mediante encuestas estructuradas y entrevistas en profundidad. Los resultados apuntan a una apropiación mediática del dispositivo impulsada por las migraciones transnacionales protagonizadas por los Saraguro en su larga historia de desplazamientos.

\title{
Palabras Clave.
}

Teléfono móvil, indígenas, kichwa-Saraguro, kipa, migraciones, comunicación

\begin{abstract}
.
Much has been written about the Saraguro Indians, however, the technological approach to these Kichw a communities, residing in the Ecuadorian inter-Andean and Amazonian zone, is practically non-existent. In this article, focusing on the mobile phone, we propose a fixed photograph of the current communicative uses of the Saraguro communities of the Ecuadorian Amazon. We selected the mobile phone because its introduction in these communities produced changes not only at an extra-community level but also intracommunity level, displacing the traditional kipa as a form of communication. Mobile phones assume these and other traditional uses of Saraguro communication, and are a paradigmatic example of media appropriation. The empirical study of this article was carried out in the south-eastern province of Ecuador, Zamora Chinchipe, between March and November 2014. The in formation was collected through structured surveys and indepth interviews. The results point to a media appropriation of the device promoted by the transnational migrations carried out by the Saraguro in their long history of displacement.
\end{abstract}

\section{Keywords.}

Mobile phone, indigenous, kichwa-Saraguro, kipa, migration, communication

\section{INTRODUCCIÓN}

Los Saraguros, comunidad indígena de etnia kichwa, residentes en el sur de los Andes ecuatorianos, han sido investigados en el marco de los estudios de etnicidad y transformaciones identitarias (Smith y Belote, 2000; Paqui y Poma, 2013; Japón, 2007; Quishpe, 2007; Anangonó, 2007; Sarango, 1995; Provencio, 2006a y 2006b; etc.). Hasta entrado el siglo XXI, los escasos estudios disponibles (firmados en su mayoría por académicos de habla inglesa, fundamentalmente norteamericanos y algunos europeos) gozaron de escasa difusión, suscitando críticas y denuncias de infraestudio de esta comunidad frente a otras similares (Sarango, 1995). Si a ello le añadimos la orientación o el interés que motivó dichos estudios, obtenemos que la aproximación a este grupo étnico desde perspectivas actuales como la tecnológica es prácticamente inexistente. 
Si rastreamos las primeras apariciones del teléfono móvil (celular) en el sur de los Andes y en la Amazonía ecuatorianos, este dispositivo no aparece en las manos de los ejecutivos o del personal docente, el asociado a la apropiación tecnológica tal y como indicó Barbero (1987). Lo encontramos, por el contrario, en las manos cuarteadas por el sol y tatuadas por la tierra arada de los familiares indígenas de los migrantes internacionales de finales del siglo XX y principios del siglo XXI. La comunicación transnacional será uno de los motores de la comunicación móvil en las regiones interandina y amazónica, pues convierte en sus primeros usuarios a los indígenas de la Sierra y la Amazonía, cuyos parientes han emigrado al extranjero. Centrándonos en un escenario $a$ priori adverso para las tecnologías, en este texto examinaremos las adaptaciones a las que ha sido sometido el teléfono móvil para cubrir las necesidades comunicativas intra y extracomunitarias de un pueblo con una larga tradición migratoria.

\section{LOS SARAGUROS: DESPLAZAMIENTOS Y APROPIACIONES}

"Las migraciones entre las/os saraguras/os no son un fenómeno reciente, sino que han formado parte de su devenir histórico" (Provencio, 2006b: 135).

Los últimos datos disponibles, los del Censo de Población y Vivienda de $2010^{1}$ del Instituto Nacional de Estadística y Censos de Ecuador (INEC), recogen la cifra de 30.183 habitantes de etnia kichwa-Saraguro en el Estado ecuatoriano. "El pueblo quichua Saraguro se encuentra asentado en un vasto territorio que, en sentido horizontal, se extiende desde el extremo noroccidental de la provincia de Loja en la región interandina, hasta las cercanías de la Cordillera del Cóndor en la provincia de Zamora Chinchipe en la región Amazónica" (Provencio, 2006a: 81). Así, los Saraguros ocupan el norte de la provincia de Loja, localizados en la ciudad de Saraguro, capital del cantón del mismo nombre, como centro neurálgico. A Saraguro, lugar que les da su gentilicio, llegaron desde Bolivia o desde Perú. Las diferentes versiones mantienen el origen de esta comunidad allén de las fonteras ecuatorianas, y los identifican con "mitimaes", los venideros, o "mitmakunas", los forasteros. Existen dos versiones de su origen. La primera se apoya en la tesis del origen peruano de los Saraguros, llegados desde el Imperio Inca por el camino del mismo nombre, el camino real del Inca. Justamente, Saraguro estaba situado en la ruta principal entre Cuzco y Quito, trazado del camino del Inca (Japón, 2007). Así, los “mitimaes”, que formaban parte del ejército real provenientes de Cuzco, se asentaron en el sur de Ecuador. La segunda versión sostiene que los forasteros, los "mitmakuna”, arribaron también de mano de los incas, en la misma época, la de su conquista de las tierras que actualmente componen Ecuador, pero no formando parte del ejército real del Inca, sino traídos por uno de ellos, el Inca Túpac Yupanqui con el fin de pacificar los pueblos conquistados. El

1. En 2010 se celebró el último censo poblacional en Ecuador, el séptimo desde que en 1950 el gobierno empezara este indicador. 
origen de los forasteros vacila, en esta versión, entre el altiplano boliviano o peruano.

Sea una u otra la crónica real de su origen, su historia pasa por el mestizaje, por los viajes y las migraciones. Movimientos que no cesan, ya que tras la llegada a Ecuador, sus desplazamientos como comunidad continúan. A finales del siglo XIX, varias familias de la comunidad Saraguro, que residían en la Sierra ecuatoriana, en el cantón de Saraguro, se desplazaron a la provincia limítrofe por el oeste, a saber; Zamora Chinchipe (Smith y Belote, 2000). Llegaron por el cantón de Yacuambi a la provincia amazónica para localizar nuevas tierras que cultivar, y se extendieron a los cantones de Zamora, Yantzaza, Nangaritza, Paquisha, El Pangui y Centinela del Cóndor (Paqui y Poma, 2013), todos situados en la Amazonía sur ecuatoriana. A estos desplazamientos internos por motivos económicos, acompañaron otras migraciones dentro de Ecuador, hacia los núcleos urbanos, en general por cuestión de estudios, o laborales. Pero también hubo migraciones externas. Los destinos de las migraciones internacionales de los Saraguros son fundamentalmente dos países, Estados Unidos y España. Dentro de este último, la región de Murcia ha sido tradicionalmente uno de los destinos escogidos por los Saraguros para vivir (Paqui y Poma, 2013). Sus continuos desplazamientos muestran que son un pueblo que tradicionalmente ha protagonizado flujos migratorios de toda índole, lo que ha propiciado un permanente contacto con otras culturas. A grandes rasgos podríamos establecer cuatro etapas diferenciadas en la historia de los desplazamientos de los kichwa-Saraguros, a saber: origen transnacional, desplazamiento interandinoamazónico, migración urbana $y$, por último, la migración internacional. La primera etapa, situada entre los siglos XV-XVI, engloba el primer gran grupo de desplazamientos y se inaugura con el mencionado viaje transnacional desde Perú o Bolivia, según las versiones, hacia el sur de Ecuador. La segunda etapa, que se abre en el último tercio del siglo XIX y se cierra a finales del siglo XX, engloba las migraciones hacia el Oriente ecuatoriano, desde la Sierra a la Amazonía. La razón principal de este desplazamiento está en el agotamiento de las tierras y el desgaste del sistema de herencias que propició el movimiento desde la zona interandina hacia los ricos valles amazónicos. Dicho con Provencio:

"La historia descrita muestra que la migración interna, especialmente hacia el oriente, se plantea tras el matrimonio como una estrategia para hacer frente a la escasez de tierras, teniendo como una de sus causas el adelanto y los patrones de herencia, que van fragmentando en exceso las propiedades familiares" (Provencio, 2006b: 138).

Esto es, desde el cantón Saraguro en la Provincia de Loja, las nuevas familias se mudaban 
a la provincia de Zamora (puerta de entrada de la Amazonía sur). Inicialmente, se instalaban en el cantón Yacuambi para buscar trabajo y así poder comprar tierras y medrar. En la sierra de Saraguro, las familias ya no podían crecer, y el ganado precisaba nuevos pastizales. De modo que la extensión natural se hizo hacia el Oriente. En un comienzo las haciendas familiares estaban conectadas, siendo las amazónicas una extensión de las interandinas. Sin embargo, en los desplazamientos de los años sesenta, se inauguró una nueva microetapa en la que las casas de la Amazonía se hiciéron autónomas, independizándose de las casas raíz en la Sierra. Las tierras de Zamora, antes de la llegada de Saraguros y mestizos, estaban habitadas por los indígenas shuar, comunidad con titularidad comunitaria de las tierras ancestrales (Vinueza, 1995).

La tercera etapa, inaugurada en los años treinta del siglo XX, tiene como origen los centros de la provincia de Loja, cantón de Saraguro (Santiago, San Lucas, Saraguro...) y como destino los centros urbanos ecuatorianos, fundamentalmente Loja, Quito y Cuenca, por motivos laborales y de estudios y nuevos cantones en la Amazonía, continuando con la búsqueda de tierras para labrar y establecerse. Desde los citados años treinta, y sobre todo desde los años sesenta y setenta llegan kickwa-Saraguros no sólo a Yacuambi sino también a los anteriormente mencionados cantones de Zamora, Yantzaza, Nangaritza, El Pangui, Paquisha y Centinela del Cóndor, en busca de nuevas oportunidades para labrar la tierra, escasa en su Saraguro natal.

La cuarta y última etapa, hasta el momento, es la de las migraciones internacionales desde finales del siglo XX y comienzos del siglo XXI, hacia España y Estados Unidos. El motor de tales desplazamientos se puede situar en el año1999, fecha en la que Ecuador sufrió el llamado "feriado bancario" que abocó a salir al extranjero a miles de ecuatorianos. Esta nueva etapa estuvo marcada por tres factores cardinales: la crisis financiera internacional, la caída del precio del petróleo y la virulencia del fenómeno atmosférico El Niño.

Se podría concluir que la convivencia con otras etnias es un fenómeno recurrente en la historia de los Saraguros. En resumen, y siguiendo a Smith y Belote (2000), conviene destacar la importancia que tuvieron y tienen las circunstancias económicas de los Saraguros a la hora de gestionar la transformación de sus identidades, tanto en origen como en las comunidades a las que se desplazan. Su posición y estatus socio-económico, aventajado frente a otras etnias indígenas del país, descansa en su fuerza económica. Los Saraguros, a diferencia de otras etnias indígenas, son propietarios a nivel individual de sus tierras de cultivo. Esta posesión individual les garantiza cierta "estabilidad con relación al resto de la población rural ecuatoriana" (Smith y Belote, 2000: 93). Sin embargo, esta búsqueda de estabilidad, traducida en la compra de terrenos, será justamente el motor de 
sus desplazamientos. Dicho con las palabras de nuestros entrevistados, los protagonistas de las migraciones de la zona interandina a la amazónica.

"Saraguro es el cantón más pobre, no hay terreno para trabajar. Allí ya no da para cultivar, por eso me he migrado para acá, como muchos de mi generación" (Entrevistado5_57años).

"En San Lucas no había recursos. No había nada. Entonces yo cogí a mis hijos y me vine para vivir aquí. Vinimos toda la familia" (Entrevistada7_57años).

"Soy venida de otra comunidad, yo vine por el trabajo. Mi comunidad donde yo nací se llama Saraguro, provincia de Loja. Hace 25 años, desde el 1991, me vine con todo (familia). Soy madre de muchos hijos. Tenía nombramiento para aquî" (Entrevistada10_65años).

Su traslado a las ciudades en las migraciones internas y externas, el cambio de matriz productiva, de la agricultura parcelaria a la agricultura-ganadería, la institución como "colonos" en Oriente (Palacios, 1999; Belote y Belote, 2000), la escolarización bilingue, y otros cambios han propiciado una serie de préstamos y apropiaciones que conviven con la permanencia de símbolos como la vestimenta. El Saraguro "mantiene su indumentaria propia ${ }^{2}$, aún en la Amazonía” (Palacios, 1999: 56). Es precisamente la capacidad de apropiación de la etnia Saraguro, el rasgo que caracteriza la historia de sus movimientos y contactos con otras culturas. Pues, justamente esta característica es la que les ha posibilitado que su nivel de conservación identitaria sea mayor a la de otros pueblos indígenas de Ecuador en circunstancias semejantes (Smith y Belote, 2000). Su capacidad de adaptar e incorporar "elementos materiales y simbólicos externos, que se articulan dinámicamente junto con los propios" (Quishpe, 2007: 61) les ha facilitado el mantenimiento de su cultura e identidad. En su historia más reciente de apropiaciones, paralela a la de sus viajes y desplazamientos, queremos destacar un elemento material externo concreto, a saber: las tecnologías en general, y su versión más puntera y actual, el teléfono móvil, en particular. En este texto, nos aproximaremos al capítulo de apropiación de las tecnologías mediáticas, sin perder la perspectiva comunitaria e identitaria.

\section{METODOLOGÍA Y TÉCNICAS DE RECOGIDA DE DATOS}

Hemos llevado a cabo esta investigación ${ }^{3}$ en la provincia sur-oriental de Ecuador

2. La indumentaria tradicional del indígena Saraguro es negra, delanas y con varias capas para resguardarse del frío serrano. Las mujeres portan enaguas o polleras y falda exterior plisada con abertura lateral y fijada a la cintura con una faja multicolor. Los hombres han variado de un juego de dos pantalones con aperturas opuestas, a un sólo pantalón negro a media caña. Desde los años treinta, los hombres visten camisas de algodón, chalecos y poncho de lana negra, y las mujeres blusas con bordados y chal anudado con un topo de plata o níquel. Ambos sexos lucen sombrero de lana prensada o borsalinos negros.

3. Para ello nos apoyaremos en los datos de un proyecto de investigación propio, Ontología Móvil y Tecno-ciudadanía nómade, financiado por la UTPL en 2014. 
-Zamora Chinchipe- empleando una metodología de triangulación, y combinando técnicas cuantitativas y cualitativas. La recogida de datos cuantitativos -mediante un cuestionario estructurado- se desarrolló entre marzo y junio de 2014. Los cuestionarios fueron rellenados en español y presencialmente, por asistentes a los talleres de tecnociudadanía ${ }^{4}$, así como por vecinos de las comunidades a las que se nos permitió el acceso como investigadoras. El estudio se realizó en siete de los nueve cantones de Zamora Chinchipe, a saber: Zamora, Centinela del Cóndor, El Pangui, Nangaritza, Paquisha, Yacuambi, Yantzaza y, más específicamente, en las comunidades de Guayzimi, Zurmi, Nuevo Paraíso, San Carlos de las Minas,San Vicente de Caney, Yacuambi, Zamora, Zumbi, La Paz y Guadalupe. Se trabajó con una muestra de 119 sujetos de entre 15 y 64 años (Ver Tabla 1). Una muestra joven -con un promedio de edad de 32,1 años, donde el $50 \%$ central está entre 20,1 y 42,1 años- y equilibrada por sexos -el 52,1\% son hombres (62) y el 47,9\% son mujeres (57)-. En cuanto al idioma, un 97,5\% afirma que habla de manera habitual español y un 38,7\% indica que emplea usualmente el kichwa, una de las lenguas, junto con el shuar-chicham, cooficiales de interculturalidad, desde que en 2008 se aprobó la actual Constitución de Ecuador.

\begin{tabular}{|l|c|c|c|c|c|c|}
\hline \multicolumn{7}{|c|}{ TABLA 1. Estadísticos de la distribución por sexo (muestra encuestada) } \\
\hline \hline Sexo & $\begin{array}{l}\text { Edad } \\
\text { promedio }\end{array}$ & $\mathrm{N}$ & $\begin{array}{l}\mathrm{Q}_{1} \\
\text { Percent. 25 }\end{array}$ & $\begin{array}{l}\mathrm{Q}_{3} \\
\text { Percent. } 75\end{array}$ & Min. & Max. \\
\hline Hombres & 30,56 & $62(52,1 \%)$ & 17,75 & 42,0 & 15 & 64 \\
\hline Mujeres & 33,67 & $57(47,9 \%)$ & 23,0 & 42,0 & 15 & 62 \\
\hline Total & 32,1 & 119 & 20,1 & 42,1 & 15 & 64 \\
\hline
\end{tabular}

\section{Fuente: Elaboración propia}

A partir de uno de los cinco apartados de los que se componía la encuesta, se obtuvo información sobre la propiedad/uso de la telefonía móvil acerca de las personas con las que los encuestados convivían. De este modo se generó una segunda muestra, o muestra ampliada, compuesta por 444 sujetos mayores de 5 años (entre 5 y 75 años) con una edad promedio de 26,7 años. De nuevo, una muestra joven y equilibrada por sexos: el 54,5\% son hombres (242) y el 45,5\% son mujeres (202). El promedio de personas por unidad

4. El acceso a las comunidades vino promovido por la realización de unos talleres en los que se reflexionaba sobre la relación entre ciudadanía y tecnologías. 
familiar es de 4 miembros, estando el 50\% central de las unidades familiares entre 2,56 y 5,14 miembros.

Atendiendo al plurilingüismo y preeminente oralidad de las personas encuestadas, elaboramos un cuestionario estructurado relativamente breve para facilitar que fuera rellenado sin demasiado esfuerzo. La encuesta se estructuró en torno a seis grandes apartados: a) datos personales, b) datos familiares, c) telefonía, d) socialización, e) medios de comunicación y f) tecno-ciudadanía. En este texto nos centraremos en los resultados obtenidos a partir de los datos recabados en los apartados b, c y f. Es decir, que abordaremos el acceso, usos y beneficios de la telefonía móvil teniendo en cuenta los datos proporcionados por los encuestados y sobre las personas con las que conviven, así como sus prácticas tecno-ciudadanas.

Los datos cualitativos, se recogieron mediante entrevistas en profundidad, realizadas entre abril y noviembre de 2014. Ante la dificultad de acceder a las comunidades indígenas, se seleccionó a las personas entrevistadas siguiendo criterios de diversidad y oportunidad, en concreto, una parte de los asistentes a los talleres impartidos. El total de personas kichwa-Saraguro entrevistadas responde al siguiente perfil: son 7 mujeres y 6 hombres de entre 15-70 años, con diferentes niveles de estudios (desde primaria a universitario), ocupadas en los siguientes sectores laborales: transportista, presidente de organización cantonal quichua, estudiante, funcionario, hostelero, auxiliar de enfermería, profesora, artesano y agricultora. En relación a la residencia, los sujetos de la muestra señalaron vivir actualmente en las comunidades amazónicas de Guayzimi, Sumakyaku, Zamora, San Vicente de Caney, Nuevo Paraíso, siendo, sin embargo, mayoritariamente, oriundos de la Sierra, de Saraguro, San Lucas y Loja.

Las temáticas del guión de la entrevista en profundidad fueron: acceso tecnológico y brechas (sexo/género, generacional, étnica, económica, educativa y lingüístico-cultural), grado de implicación comunitaria/política, usos tecnológicos, tradición-modernidad, historia comunicativa y socialización digital. Todas las entrevistas fueron grabadas y transcritas, y realizadas en español, sin intérpretes.

\section{ENTRE KIPAS, CHASQUIS Y TELÉFONOS MÓVILES}

\subsection{De la Kipa al móvil: comunicación intracomunitaria}

Los sonidos en las comunidades kichwa-Saraguro marcan los ritmos de la cotidianidad de pequeñas haciendas dispersas en la Sierra y más agrupadas, por lo general,en la Amazonía ecuatoriana. Los gallos visualizan la ruptura del alba y la hora del primer ordeño, los grillos anticipan los chaparrones tropicales, las llamadas de la kipa, o en la actualidad los agudos de los altoparlantes colocados en el local social convocan a los vecinos a una minga para recibir las fiestas y los bips de los mensajes, politonos y temas musicales descargados en el móvil indican que la jornada finalizó o que los adultos han 
vuelto de la chacra, la huerta, para cenar y están descansando, en las canchas deportivas o sentados delante de la puerta de sus casas de hormigón y madera. De entre todos estos sonidos, dos son intencionales, los derivados de la kipa y el móvil, los dos principales medios de comunicación de los kichwa-Saraguros en su pasado y presente.

La kipa es un cuerno de vaca tratado artesanalmente que se utiliza como instrumento y que emite un sonido similar a un pitido:

"La comunicación tradicional con la kipa, el cacho de res grande. Eso funcionaba en los pitos para llamar para una minga, una reunión, una fiesta, etc. Eran diferentes pitos. Yo he utilizado la kipa. Hace 20 años que no lo veo. Ya no me acuerdo bien del código. No tocaba cualquiera. El que quería aprendía a tocar, si no no" (Entrevistado5_57años).

El desuso de la kipa, coincide en los años noventa con la introducción de la telefonía celular en Ecuador y también con el inicio de la cuarta etapa de desplazamientos migratorios del pueblo Saraguro, a la que antes hacíamos referencia. La llegada de los móviles vendrá de la mano de una generación de migrantes internos desplazados a la capital y a las grandes ciudades del país, pero sobre todo de los emigrados externos a destinos internacionales, como España y Estados Unidos. Es el caso de varios de nuestros entrevistados, quienes recuerdan haber visto un celular por primera vez en manos de familiares que los traían de la emigración. Las primeras ganancias en el viaje migratorio se empleaban en adquirir un terminal telefónico móvil para poder mantener contacto con la comunidad de origen.

“A mi papále vi el primer celular, hace ya más de diez años. Eran los celulares que recién salían, los que por primera vez salían eran... Lo compró para comunicarse con la familia que se encontraba en el extranjero" (Entrevistada3_18años).

"Yo por teléfono hablo con mis hijas que pasan en España. Hablamos con ellas, pero si les digo yo en kichwa ellas no entienden lo que les digo" (Entrevistado11_70años).

"Ya existe la interacción entre muchos compañeros, de muchos lugares, de diferentes provincias e incluso de otrospaíses, pues, ya conocían más la tecnología. Entonces, uno también ya se ha hecho algunos esfuerzos para conseguir ese tipo de teléfonos" (Entrevistado1_26años).

O en sus propias manos, siendo ellos los protagonistas de estos flujos migratorios transnacionales con sus consecuentes cambios comunitativos:

"Yendo a España compré un celular (...) Aquí no utilizábamos nosotros todavía, vivíamos aquí en el campo (...) Cuando fui para allá me hizo mucha falta para comunicarme con la familia (...) Ahora todo el mundo utiliza. Nos hace falta para comunicar. Estamos regados por un lado y otro, por todos lados. El 
celular es muy importante. Tenemos familiares en España, en Estados Unidos. Cualquier cosa, nos llaman. Es muy útil”' (Entrevistado5_57años).

"Me fui a España. Ahí, primera vez me compré un celular. Era una herramienta súper interesante para trabajo mismo. Facilita para llamar, hacer contactos. Utilicé un tiempo. Estaba por dos años y medio en España, en la ciudad de Marbella. Retorné y como no hay aquí la señal no se ha utilizado. En los tiempos, antes, había que salir cada dos o tres meses de este lugar a la ciudad y no era interesante invertir en un celular. Y sobre todo, no hace mucho tiempo que esto del celular se dio ya a nivel nacional en nuestro Ecuador. Solo en las partes capitales, Quito y Guayaquil, manejaban el sistema. Pero a la parte de la provincia de Zamora se desconocía. Ahora, poco a poco, se está avanzando y nos facilita la comunicación. Al menos aquí todavía estamos aislados con la cobertura celular. Es algo complicado. Al menos aquí teníamos telefonía comunitaria, un tiempo nos dio buen servicio, pero ahora está averiada. Lo han querido reparar: la parte técnica. Todavía no lo han logrado. Dicen que son fallos del sistema de satélite. Pero en ello todavía nos facilita esto del Internet. Estamos utilizando vía Internet las comunicaciones, a través de chat, de correo, Facebook, eso" (Entrevistado13_35años)

Así, Saraguro y las zonas de asentamiento de los kichwa, protagonistas de las migraciones internacionales de los años noventa, comparten con las grandes ciudades de Ecuador, Quito y Guayaquil, el mérito de ser los primeros lugares donde se vieron los teléfonos móviles.

"En Saraguro (vio el primero). Allí si hay cobertura. Más antes solo eran sencillos, ahora ya tienen cámaras, fotos, Internet, más modernos" (Entrevistada12_32años).

La migración generó una entrada significativa de teléfonos móviles: "no le podría decir cúal fue el primer celular que vi, pero igual de pronto, así todos empezaron con los celulares..." (Entrevistada9_45años). Dicha entrada fue posibilitada por la necesidad de la comunicación transnacional. Necesidad que, como indica la siguiente entrevistada, cubren las Tecnologías de la Información y la Comunicación (TIC), en general y el teléfono celular, en particular: "Lo mejor de las tecnologías es que nos podemos comunicar con alguien que está lejos de aquí, como Estados Unidos,que no se puede ir a ver y nos comunicamos por Redes Sociales" (Entrevistada8_16años).

Nuestros datos cuantitativos revelan que un 57,1\% de los kichwa-Saraguros residentes en la Amazonía sur son propietarios de celulares, reflejando una diferencia significativa por sexos, ya que si bien el 62,9\% de los hombres tienen teléfono móvil en propiedad, sólo 
un 50,9\% de las mujeres está en la misma situación, evidenciando una brecha de sexo/ género que se yuxtapone a la de etnia (Martínez, de Salvador y de Salvador, 2015).

En lo referente a la edad, debemos destacar que se trata de jóvenes, ya que la edad promedio de los propietarios es de 32,3 años frente a 31,7 años de los no propietarios. Lo que explica el dato de antiguedad respecto de la apropiación de un celular pues los kichwa-Saraguros amazónicos tienen un teléfono móvil desde hace 32,5 meses, estando situados los extremos en diez años de propiedad y en sólo un mes desde la compra del primer dispositivo.

De entre los no propietarios (el 42,9\% de los encuestados), un 70,6\% indica que sí usa teléfono celular, pero en préstamo. Lo que implica un aumento considerable en el acceso a los dispositivos móviles. Así, de todos los que usan celular (el 87,4\%), el 34,6\% lo usan prestado por un familiar, un vecino o un amigo.

Los encuestados además de facilitarnos datos propios, nos ofrecieron información relativa al acceso a los celulares (en propiedad o préstamo) de personas con las que convivían. De ahí obtuvimos una muestra ampliada, cuyos datos reflejan variaciones con respecto al acceso en función del sexo y la edad frente a la muestra reducida. El dato más dispar es la diferencia en la edad promedio entre propietarios ( 31,2 años) y no propietarios $(23,6$ años) (Ver Tabla 2).

TABLA 2. Propiedad de dispositivos móviles por sexo con edad promedio (muestra ampliada)

\begin{tabular}{|l|l|l|l|l|l|}
\hline $\begin{array}{l}\text { Tiene móvil } \\
\text { (propietarios) }\end{array}$ & N & \% total & $\begin{array}{l}\text { Edad } \\
\text { promedio }\end{array}$ & $\%$ hombres & $\%$ mujeres \\
\hline NO & 264 & $59,5 \%$ & 23,6 años & $59,1 \%$ & $59,9 \%$ \\
\hline SI & 180 & $40,5 \%$ & 31,2 años & $40,9 \%$ & $40,1 \%$ \\
\hline N Total & 444 & & 26,7 años & 242 & 202 \\
\hline
\end{tabular}

\section{Fuente: Elaboración propia}

Con respecto al sexo, no existen diferencias notables en la propiedad de celular. Sin embargo, sí existen diferencias significativas por sexo en su uso. Es decir, hay disparidad entre aquellas personas que sin ser propietarias del dispositivo sí son usuarias de la telefonía móvil (ver Tabla 3). Esto es, del total de las personas de la muestra que indican no tener en propiedad un teléfono móvil, el $40,5 \%$ usan uno prestado. Las mujeres que no 
tienen móvil lo usan prestado en mayor porcentaje que los hombres, esto es, son usuarias en préstamo el $47,1 \%$ de las mujeres frente al $35,0 \%$ de los hombres no propietarios pero sí usuarios (diferencia de proporciones: $\mathrm{z}=2,01 ; \mathrm{p}=0,05$ ).

TABLA 3. Usuarios en préstamo no propietarios (muestra ampliada)

\begin{tabular}{|c|c|c|c|c|}
\hline Sexo & No tiene móvil & Usa en préstamo & $\%$ & $\begin{array}{c}\text { Diferencia de } \\
\text { proporciones }\end{array}$ \\
\hline Hombres & 143 & 50 & $35,0 \%$ & \\
\hline Mujeres & 121 & 57 & $47,1 \%$ & $\begin{array}{c}\mathrm{d}=12,1 \% \\
\mathrm{Z}=2,01 \\
\mathrm{p}=0,05\end{array}$ \\
\cline { 1 - 3 } Total & 264 & 107 & $40,5 \%$ & \\
\hline
\end{tabular}

Fuente: Elaboración propia

Este representativo dato sobre el uso del préstamo refleja un contexto de fuertes lazos comunitarios. Los teléfonos móviles, para Lasén (2009), representan una "extensión de la presencia" y "contribuyen a la formación y mantenimiento de lazos sociales y vínculos interpersonales". Este análisis tomaría más relevancia en nuestro contexto, con la lógica de los préstamos, puesto que los móviles perderían parte de su sentido individual ${ }^{5}$.

Frente a los teléfonos móviles, utilizados por ambos sexos, la kipa, como medio de comunicación, era una herramienta de convocatoria, con un código compartido por los miembros de la comunidad que usaban exclusivamente hombres. Tanto a nivel familiar, los "padres de familia" para llamar a los vecinos, como a nivel comunitario, los líderes para convocar a todo el pueblo a asambleas o mingas ${ }^{6}$. En palabras de los protagonistas:

"La kipa se maneja a nivel de comunidades. Si la comunidad está organizada ya tiene la contraseña. Si hay un problema se saca la kipa y la sociedad ya sabe que hay un problema y se reúnen. Había una persona encargada para eso, para tocar la kipa. Nuestros abuelos acostumbraban a tener en cada casa... La tocas

5. La individualidad asociada a los teléfonos móviles frente a los fijos, de uso familiar, pivota en parte en la posibilidad de personalizar su carcasa pero también en rellenar su memoria interna con politonos, fotografías, números en la agenda, etc. Los casos de préstamo que hemos encontrado en los indígenas kichwa-Saraguros son fundamentalmente de dispositivo con chip incluido, lo que deja un margen irrisorio a la individualidad enunciada de quien lo usa en préstamo. Sin embargo, también hemos conocido casos en los que se prestan los dispositivos sin el chip, y cada usuario coloca su propia SIM lo que al menos permite el poder responder de modo individual y portar la agenda.

6. Las mingas son jornadas de trabajo comunitario en las que cada cual contribuye con sus habilidades y se hacen intercambios para suplir contrataciones de servicios a personal externo. 
y vienen todos a la casa. El padre de familia tocaba" (Entrevistado6_31años). "La kipa era para llamar a la minga. Había una gente dedicada de propia decisión, de propio interés a tocarla. Hasta donde yo sé sólo hombres" (Entrevistada7_57años).

"Si aquí no más necesitamos hacer algo, el cacho de la vaca (la kipa) se tocaban durísimo y todo el mundo escuchaba y venía a ver que estaba pasando. Ahora ya no se utilizan. Pero también estamos por recuperar esas cosas" (Entrevistada9_45 años).

"Para llamar a la comunidad era la campana o el cacho. Para las mingas era el cacho. Había un señor encargado, el mayoral. Cuando ya se forma la directiva, ahí entran en la directiva, el presidente, secretario, tesorero más los vocales, el mayoral y el síndico. El mayoral siempre eran hombres, no sé por qué, hombres eran" (Entrevistada10_65años).

"Aquí (mingas de limpieza) hacemos. Ahora estamos haciendo muy lentos, más antes sabíamos hacer casi cada seis meses la limpieza de las comunidades. Hacemos una sesión con toda la gente. Una sesión general, y en esa nos quedamos de acuerdo para hacer tal día la minga. Las convocatorias nosotros hacíamos, ahoritas no tenemos aquí, porque se nos llevaron, por medio de una kipa o cacho de vaca, se le suena, la gente ya sabe para qué y por qué. Teníamos una kipa. Hace un año que la fueron llevando para una reunión que hicieron por abajo, por Yantzatza, pero por ahí creo que le habían quitado los policías o los militares. De ahí no tenemos. Pero vamos a volver a hacer de nuevo. Si lo podemos hacer nosotros. Eso lo han sabido hacer los antiguos, los viejitos. Pero también lo podemos hacer" (Entrevistado11_70años).

"(Mi madre) dice que no había nada, antes no había nada, tenían que ir ellos mismos a comunicar. Después ya había unos cables de telegramas, después ya habían los teléfonos. Con esa (la kipa) ellos le kipaban, le comunicaban a la comunidad. Yo los vi, porque en Saraguro siempre es el cacho que tocaban, ahoritas ya es el micrófono. De ahí, más antes era solo el cacho, donde yo vivía con mi mamá. Es de la comunidad, el que decían el cabildo, el que tocaba. Cuando había mingas ya le tocaba el cacho y los que ahora es de salir a la minga, como una sirena que es" (Entrevistada12_32años).

La kipa es hoy en día un instrumento casi en desuso. Ya pocas quedan en las comunidades, como explica el anterior entrevistado que narra la incautación de la última de la comunidad San Vicente de Caney. Su rol de convocatoria, compartida en tiempos con la campana, lo asumen hoy nuevos instrumentos, entre los que destaca la bocina o altavoz, muy útil en comunidades con poca o nula señal de cobertura e incluso el móvil. Este salto es narrado 
por los propios kichwa-Saraguros amazónicos, quienes recuerdan el paso de uno a otro instrumento en su función de comunicación o convocatoria intracomunitaria.

"A veces era la campana. Aquí hay la bocina, el altoparlante, cualquier cosa, cualquier actividad que tienen que hacer en la comunidad entonces es por el altoparlante. El altoparlante ya lo prenden a cualquier hora. Todo rápido. Ya está dicho. Hay que reunirnos cuando llaman por el micrófono" (Entrevistada10_65años).

"Ahoritas tenemos un altoparlante en sitio bien alto. Siempre para una sesión convocan por medio del altoparlante y ya está. Con celular (se comunican para las convocatorias) tal vez a algunas personas con los que están de acuerdo, pero no con todos, no hay cómo. Por ejemplo aquí no tenemos cobertura, muy poca cobertura. Hay veces que tenemos que salir a diferentes partes para ponernos en contacto con otros lados" (Entrevistado11_70años).

Casi la mitad de los kichwa-Saraguros amazónicos encuestados, un 48,6\%, manifiesta haber empleado un teléfono celular para una convocatoria de acto público, esto es, para llamar a alguien y anunciarle una reunión, asamblea o acto comunitario. El porcentaje se reduce al $28,4 \%$ para quienes han participado de convocatorias por la Red y a un $23 \%$ para quienes lo han hecho mediante un SMS o similar (Ver Tabla 4).

\begin{tabular}{|c|c|c|c|c|}
\hline \multicolumn{5}{|c|}{ TABLA 4. Frecuencias Tecno_ciudadania } \\
\hline \multirow{2}{*}{\multicolumn{2}{|c|}{ Tecno_ciudadania ${ }^{a}$}} & \multicolumn{2}{|c|}{ Respuestas } & \multirow{3}{*}{$\begin{array}{l}\text { Porcentaje de } \\
\text { casos } \\
85,7 \%\end{array}$} \\
\hline & & \multirow{2}{*}{$\frac{N^{\circ}}{36}$} & \multirow{2}{*}{\begin{tabular}{|c} 
Porcentaje \\
$48,6 \%$
\end{tabular}} & \\
\hline & TC_cel acto publ & & & \\
\hline & $\begin{array}{l}\text { TC_SMS } \\
\text { asociacion }\end{array}$ & 17 & $23,0 \%$ & $40,5 \%$ \\
\hline & $\begin{array}{l}\text { TC_comun asoc } \\
\text { internet }\end{array}$ & 21 & $28,4 \%$ & $50,0 \%$ \\
\hline Total & & 74 & $100,0 \%$ & $176,2 \%$ \\
\hline
\end{tabular}

\section{Fuente: Elaboración propia}


La falta de señal móvil o de "cobertura intermitente" (de Salvador y Martínez, 2015a), así como de señal de Internet, es quizás una de las causas de estos bajos porcentajes de uso del celular y de Internet para la comunicación intracomunitaria, en el caso concreto de convocatorias públicas. Es precisamente la falta de cobertura la que diversifica los usos del teléfono por parte de los usuarios kichwa-Saraguro. Así además del uso comunicativo intracomunitario o extracomunitario, los teléfonos móviles son aprovechados en las comunidades amazónicas kichwa-Saraguro como cámaras, linternas o reproductores musicales. Este último uso, refuerza al móvil como sustituto de la kipa, como instrumento relacionado con las festividades. Esto es así, ya que las fiestas comenzaban con el toque de kipa para convocar a la minga de limpieza. Los sonidos del cacho anunciaban y acompañaban las principales citas de los vecinos en las celebraciones de la comunidad. Los entrevistados enumeran algunos de los usos no comunicativos que le dan a sus celulares:

"Mi primer celular es el Nokia 1100. Sí, que más se utilizaba como linterna, más que para otras cosas. Como reloj, como despertador, como linterna y despertador “(Entrevistado1_26años).

"Sirve como cámara, para música dispersión... como uno lo use y de acuerdo al equipo que tenga” (Entrevistado6_31años).

"Lo uso para la música y para llamar también" (Entrevistada8_16años).

"Los jóvenes tienen más celulares, porque a ellos les gusta la música, vídeos... Música, Internet” (Entrevistada12_32años).

"El celular me sirve para escuchar música, ver el calendario mismo, algunas que se guarda algunas notas en la agenda. Sirve como de linterna, a veces se va la luz. Nos sirve sin cobertura. La línea telefónica más cercana está en las Orquídeas, viajando una hora. No hay cobertura celular pero se puede hacer una llamada de un convencional, porque nos facilita un poco. De ahí ya hablando de la cobertura celular tenemos en el cantón Nangaritza, son casi tres horas, en Guayzimi. Ahí ya tenemos cobertura. Sale costosa ya la llamada porque el pasaje y todo eso ya..." (Entrevistado13_35años).

Así, un 87, 4\% de los kichwas de la muestra simple manifiestan ser usuarios del teléfono móvil con los siguientes usos frecuentes (Ver Gráfico 1). Cada sujeto marcó un promedio de 4,7 usos: llamar es la función que usan en mayor medida, seguida de reproducir música, y, en menor uso, las descargas (tonos, juegos y fotos). La falta de cobertura $3 \mathrm{G}$ o 4G, así como la obsolescencia de los dispositivos usados, pues muchos de ellos no tienen acceso a Internet, explicarían estos datos. 


\section{GRÁFICO 1. Porcentaje de los usos del móvil}

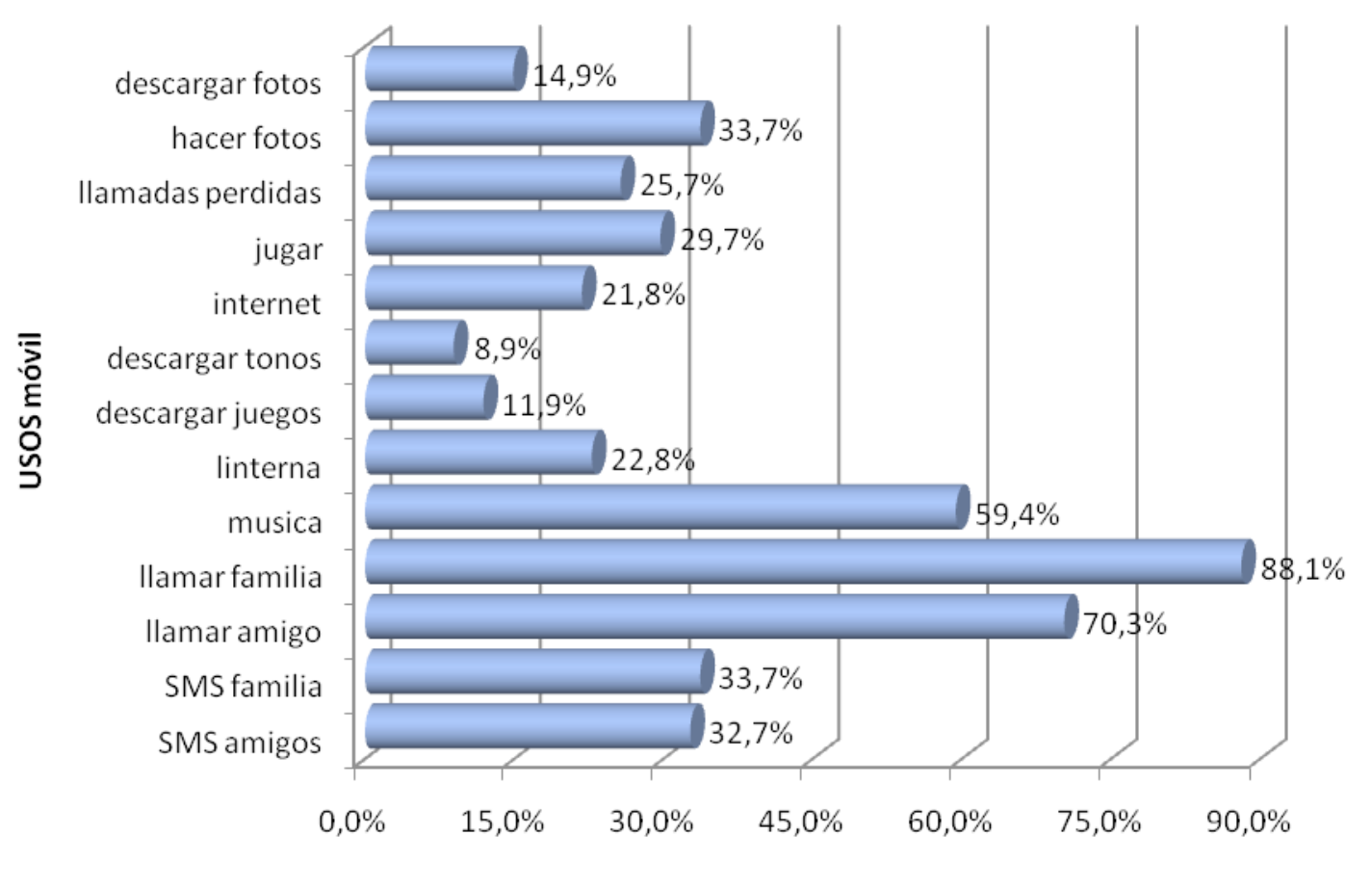

\section{Fuente: Elaboración propia}

Atendiendo a la muestra simple, únicamente el 30,8\% tiene Internet-wifi en su dispositivo, con una diferencia de más de diez puntos si desglosamos los datos por sexo, ya que el $35,7 \%$ de los hombres que usan móvil tienen Internet, frente al 25,0\% de las mujeres (si bien estas diferencias no son significativas en la población para un NC del 95\%).

Igualmente, la edad promedio de hombres y mujeres que usan móviles con Internet no son significativamente diferentes (prueba $U$ de Mann-Whitney: $Z=-0,176 ; p=0,860$ ).

Si nos centramos en los usos que hacen los kichwa-Saraguros amazónicos que acceden a la telefonía móvil a partir de dispositivos con Internet o tecnología Wi-fi obtenemos que el uso mayoritario es la música con un 14,2\%, seguida de las llamadas a familiares y amigos (11,8\% en cada caso), navegar por la Red $(8,8 \%)$ y mandar SMS a amistades $(8,3 \%)$ y a la familia $(7,8 \%)$.

Si otrora la kipa convocaba a la comunidad con su sonido para reuniones o mingas, mostrando su fuerza como herramienta mediadora intracomunitaria, hoy, los teléfonos móviles, el nuevo medio de comunicación apropiado por el pueblo kichwa-Saraguro, cubrirían no solo las necesidades comunicativas intracomunitarias, y sustituirían por tanto el uso de la kipa, sino que satisfarían también las necesidades de la comunicación extracomunitaria, en la que las comunicación transnacional juega un papel fundamental. 


\subsection{Aproximación a la historia de una apropiación: de chasquis y teléfonos móviles}

El teléfono móvil, además de hacer frente a las necesidades de comunicación intracomunitaria, como antes hizo la kipa, cubre también las necesidades de comunicación fuera de la comunidad, ampliando así el rango de acción de los contactos que permitía el instrumento de comunicación intracomunitario tradicional. Consciente de la rapidez de los cambios, uno de los entrevistados apunta a cómo las tecnologías están penetrando en las comunidades y sustituyendo al tradicional chasqui, al mensajero, al portador de noticias, al Hermes griego que, en la cultura inca, recibía el nombre de chasqui o chaski:

"Antes para comunicarnos teníamos las cartas, el chasqui, la noticia. El chasqui es que alguien llevaba la noticia a entregarle. Una persona encargada. Traía el mensajero. Era como un síndico de la comunidad. Siempre hombres" (Entrevistada9_45 años).

"Muy, muy diferente todo era allá, por ejemplo, ahora mismo, en el momento de convocar. Todo era que alguien tenía que dejar un oficio, alguien tenía que llegar allá. Ahora mismo, a veces, teníamos que enviar un oficio por la Cooperativa Zamora, por tal Cooperativa de transporte. Pero yo, ahora, simplemente redacto una convocatoria y envío directamente a otra persona, listo. Y, a veces, solamente les pego un toque y reviso mi correo, que ya envié y ya está, listo. Entonces, no es necesario que de aquí tengo que llegar a Guayzimi, que tengo que enviar en cooperativa de transporte, en fin”(Entrevistado1_26años).

"Como presidente de la asociación, luego de conocer el tema ya les paso unos comunicados, algunos enlaces por Internet, a los compañeros, un manifiesto. También que muchos de los compañeros de las organizaciones, de hecho la mayoría, aunque sea uno, dos, tres, cuatro, cinco integrantes, ya manejan este tipo de tecnologías. Claro que no tienen quizá un celular, pero tienen acceso, en colegios, en algún lugar. Entonces, y a veces yo les comento, les paso esto, incluso para las convocatorias y les decía simplemente, les escribo una convocatoria en Word, en lo que sea, y luego ya les subo, leen el documento para tal fecha, entonces, ya estamos en contacto" (Entrevistado1_26años).

"Para la parte de afuera lo utilizamos el Facebook y el correo, en la parte local utilizamos una redacción de escritos porque no hay comunicación, no tienen otras comunidades estas redes sociales para poder comunicarse, solo a través de palabras o documentos escritos se les hace llegar una invitación o comunicación" (Entrevistado13_35años).

Como puede colegirse de las palabras del entrevistado la kipa o el chasqui ya sólo tienen espacio en aquellos lugares a donde las tecnologías no llegan. Podríamos destacar aquí dos posibles causas de la denominada como infopobreza, en lo referente al acceso de 
la tecnología móvil en la zona: la causada por la ausencia de recursos económicos de los usuarios a la hora de comprarse el terminal y recargarlo, y la causada por la falta de infraestructura estatal que garantice la cobertura en la Amazonía. Si comparamos las cifras con otras zonas del país (de Salvador y Martínez, 2015b), ambas causas son responsables del bajo nivel de penetración móvil en las comunidades indígenas de la Amazonía. Sin embargo, ante esta situación los kichwa-Saraguros afectados ingenian una serie de estrategias para paliar su falta de acceso, entre las que destacan el préstamo de celulares para combatir la carencia de terminales y la persona a modo de teleoperador encargada de atender la llamada, la localización de las lomitas con 3G y los compartidos con bluethooth entre otras estrategias para superar la falta de cobertura (de Salvador y Martínez, 2015a).

La primera de las barreras de acceso a la telefonía móvil, la económica, es refrendada por la información obtenida en nuestra investigación. Los datos de uso y propiedad en relación con la edad de los propietarios y usuarios refuerzan esta tesis. Además, si desglosamos la información de acceso por sexo, esto es, si comparamos los datos de los propietarios y usuarios, en función de si son hombres o mujeres, observamos que ellas sufren una serie de brechas (Martínez, de Salvador y de Salvador, 2015). En las entrevistas, el primer teléfono móvil llega a los usarios, en su mayoría, con el sueldo del primer trabajo en la emigración urbana e internacional, interna y externa. Los entrevistados dicen que para acceder a la compra de un celular es necesario tener un trabajo, y que su adquisición redunda en beneficios laborales, o dicho de otro modo, que son necesarios para trabajar.

"Ahora mismo, por ejemplo, le puedo decir quienes tienen celular son las personas que están trabajando fuera de la casa, fuera del pueblo, pero los que tienen acceso a estas tecnologías, por ejemplo, a las redes sociales, específicamente son más los estudiantes, son más jóvenes que están en el colegio, y que por diferentes razones, ya sea porque el docente les manda a investigar, o a veces, porque uno se quiere también ingresar al mundo de las tecnologías, ellos acceden a un Internet" (Entrevistado1_26años).

"Hay más gente que lo tiene porque ahora lo pueden comprar, y si es que no lo pueden manejar bien, igual lo podemos enseñar y pueden también manejar" (Entrevistada3_18años).

Los kichwa-Saraguros amazónicos entrevistados son conscientes de que se trata de una cuestión de clase, de origen rural (Bidwell et. al, 2011).

"La clase social, eso, es primordial. La clase social es lo más importante, porque si vamos a una comunidad que no hay muchos ingresos económicos, no podemos usar, por ejemplo, uno de estos celulares (smartphone). Sí podemos usar, por ejemplo, un Nokia simplemente, a veces, solamente para poder llamar y recibir 
llamadas, mensajes, y a veces como linterna. Pero ya así para tecnología, para estar al tanto de las noticias, eso no. Entonces, yo creo que sí es muy importante la clase económica, la clase social" (Entrevistado1_26años).

"La tecnología en general es extremadamente buena utilizándola bien. Al mismo tiempo se necesita un poco de capacitación en el tema de los pueblos y nacionalidades. No estamos aislados, más bien estamos un poco distanciados por el tema geográfico, y por problemas socioeconómicos. Eso a veces nos limita a estar al día, al tanto. La gente que está en las ciudades si está al día... En cambio el campo es diferente. Se necesita un programa de capacitación para la inserción de los pueblos y nacionalidades al manejo de tecnologías para poder interrelacionarse entre pueblos y nacionalidades y buscar acercamientos con la sociedad en general, nacional y de fuera del país" (Entrevistado6_31años).

Asimismo, son conscientes, que las diferencias de clase están atravesadas por las brechas de sexo/género en el caso de las mujeres.

"Será precisamente porque, a veces, los hombres como que nos dan ya más responsabilidades, o porque, a veces, el tema de, quizá un celular no nos compran nuestros padres, sino, a veces, nos toca comprar nosotros mismos, y a veces, para comprarlo ya lo hacemos por una razón, en cambio, el celular para una chica, lo compran sus padres y quizás no lo saben valorar mucho" (Entrevistado1_26años).

"Ellos necesitan teléfono móvil. O, a veces, cuando la esposa se dedica solamente a la casa, como que, no le hace mucha falta el celular. Pero, en cambio, si su situación es de trabajo, es donde más se utiliza el celular"(Entrevistada3_18años).

"Nunca tuve celular. Necesito de dinero. Son caros. Nosotros no tenemos ni finca, ni nada. Mis hijos como ya entraron a trabajar ya tienen su dinerito y tienen para el celular, la casa y todo" (Entrevistada7_57años).

"Tienen celular los mayores y los hombres. Tienen más que las mujeres porque alli hay pocas mujeres, y ellas tienen algunitas celular pero menos que los hombres" (Entrevistada8_16años).

"Tenía mi esposo celular, no he comprado yo. Cuando voy por ahí me voy llevando el de mi esposo. Hace un año que tiene pero recién le botó, mi esposo" (Entrevistada12_32años).

"Parece que tienen más celulares los hombres. Los que son como, se le utiliza como un medio de gestión, para averiguar alguna información. Sí he visto más en la parte adulta. En la parte joven son las dos partes que están manejando. No le he analizado la situación, pero yo veo que la mayor parte es más el 
hombre lo está manejando el sistema. Poca importancia le dan, se manejan la información a través de... Las que tienen marido le piden al marido, prestado. Sobre todo a lo mejor le digo por lo que no hay esa cobertura para hacer utilidad personalmente. Entonces un celular a veces se maneja en la casa, en la familia, no se está manejando casi como uso personal, en el caso de este lugar" (Entrevistado13_35años).

A la hora de comprarse un teléfono celular los Saraguros amazónicos intentan satisfacer dos necesidades, la de abaratar y hacer más cómoda y posible la comunicación, con familiares y amistades, y la de favorecer sus oportunidades laborales. Así lo reflejan tanto los datos de las encuestas, como sus testimonios. Casi la mitad de la muestra simple indica que el motivo para comprarse un teléfono móvil es primordialmente que ahorra tiempo y dinero, seguido del beneficio laboral que supone adquirir uno (Ver Tabla 5).

TABLA 5. Beneficios Promedio

Usan celular $=104$, contestan 96 . Marcan como promedio 1,6 beneficios.

\begin{tabular}{|c|c|c|c|c|}
\hline \multicolumn{5}{|c|}{ beneficios frecuencias } \\
\hline \multirow[b]{2}{*}{ beneficios ${ }^{\text {a }}$} & & \multicolumn{2}{|c|}{ Respuestas } & \multirow{2}{*}{$\begin{array}{r}\begin{array}{r}\text { Porcentaje } \\
\text { casos }\end{array} \\
\end{array}$} \\
\hline & & $\mathrm{N}$ & Porcentaje & \\
\hline & Benef_mas seguro & 15 & $9,7 \%$ & $15,6 \%$ \\
\hline & Benef_moda & 6 & $3,9 \%$ & $6,3 \%$ \\
\hline & Benf_comod_barat & 70 & $45,5 \%$ & $72,9 \%$ \\
\hline & Benef_trabajo & 39 & $25,3 \%$ & $40,6 \%$ \\
\hline & Benef_musica & 24 & $15,6 \%$ & $25,0 \%$ \\
\hline & Total & 154 & $100,0 \%$ & $160,4 \%$ \\
\hline
\end{tabular}

Fuente: Elaboración propia

Los protagonistas amplían, en las entrevistas, las razones para comprarse un celular por cuestiones laborales:

"Tengo el celular para comunicarme con mis hijos. Están fuera, estudiando, el otro trabaja en Cuenca, otros en Saraguro, otros en Zamora, así dispersos (...) Me da vergüenza decir. Tengo 12 hijos. Todos tienen celular. El último hijo tiene 
20 años. Yo llamo. A veces ellos me llaman"(Entrevistada10_65años).

"Finalizando la secundaria, tuve la oportunidad de salir a diferentes lugares, en primera instancia a trabajar. Uno, como joven, a esa edad sale, supuestamente, a trabajar o a hacer dinero, entonces, en ese momento, pues, es que compro mi primer celular (...) sí, para el trabajo" (Entrevistado1_26años).

"Tengo celular desde los 17 años, cuando recién llegaron a Ecuador. El primero fue por vanidad, con el pasar del tiempo pasó a ser una herramienta de trabajo, y hoy más que nunca, no puedo dejar mi teléfono" (Entrevistado6_31años).

"El primer teléfono me lo regaló mi jefe. Porque antes de los teléfonos había el busca, se llamaba a una central y la central le mandaba el mensaje. Pitaba" (Entrevistado6_31años).

El motivo de la seguridad o de rapidez de reacción frente a las emergencias o accidentes, señalada como el principal beneficio a la hora de comprarse un celular por el 9,7\% de los encuestados, es también mencionado en las entrevistadas:

"No tengo yo pero veo. Me emociona es ver rapidito para poder comunicar cualquier cosa de un lado a otro, malo o bueno o lo que sea, pero está excelente. Yo si veo que es importante. Para una enfermedad de eso se salva rapidito porque las comunicaciones largas llegan" (Entrevistada7_57años).

"Caso que se han dado de súper urgencias, urgencias en calamidad doméstica, la salud. A veces hemos intentando comunicar para pedir una ambulancia y no ha habido ni la posibilidad ni a través de Internet. No están conectados. Lamentable. Si tuviéramos una apertura de celular llamaríamos en cualquier momento que se necesitara" (Entrevistado13_35años).

\section{CONCLUSIÓN}

La apropiación de la telefonía móvil en las comunidades kichwa-Saraguro de la Amazonía ecuatoriana viene de la mano de una serie de desplazamientos protagonizados por este grupo étnico. Su sistema de herencias y de agricultura parcelaria pivotada sobre la propiedad individual de las tierras es lo que marcará su reciente etapa de migración hacia la Amazonía sur, como parte de su cadena de estrategias adaptativas (Palacios, 1999). La capacidad de adaptación que acompaña y acompañó su larga historia migratoria se pone así mismo de manifiesto ante un objeto externo, como el teléfono móvil, en un contexto amazónico adverso para su uso. Así, tal y como nos han contado los propios protagonistas, los préstamos tecnológicos, y en particular los celulares, se han ido incorporando a la rutina diaria de las comunidades. En algunos casos supliendo formas ancestrales de comunicación intracomunitaria y, en otros, ampliando el rango de contacto y acción extracomunitario, fundamentalmente en lo relativo a las relaciones con 
parientes emigrados a España o Estados Unidos. Así, en el primer sentido, se ha dado el salto de la kipa al móvil, esto es, de un instrumento tradicional -en manos exclusivamente de los hombres para convocar a la comunidad, tanto para asuntos de carácter político como festivo- al móvil como herramienta para mantener los lazos comunitarios. Si bien persisten en el acceso al móvil brechas de sexo/género motivadas por una clara cuestión económica, su uso parece responder a una cuestión etaria. Son fundamentalmente los más jóvenes de las comunidades quienes ahora emplean los móviles y refuerzan los vínculos interpersonales. En un segundo sentido, la entrada del dispositivo móvil aparece ligada a los mencionados desplazamientos, pues será la necesidad de comunicación trasnacional la que demande el contacto con las comunidades de origen de sus parientes desplazados internacionales. Si antes el mensajero, el chasqui, era el encargado de portar un mensaje recurriendo a los medios de transporte tradicional, ahora el móvil suple su función, abaratando y facilitando el contacto, principal motivo que aducen los kichwas-Saraguro para comprarse un celular. Pero igualmente aquí nos encontramos con desigualdades y dificultades en su acceso motivadas, ahora, por un contexto desfavorable para la red telefónica y el acceso al propio dispositivo. A pesar de ello, su uso, tal y como hemos visto, les lleva a ingeniar estrategias de apropiación que siguen fortaleciendo su capacidad de ocupar su espacio fuera de su lugar natal, escribiendo así un nuevo capítulo en su historia de adaptaciones y supervivencias. 


\section{REFERENCIAS BIBLIOGRÁFICAS}

Anangonó, Emilio (2007) "La identidad para los Saraguros, una necesidad fundamental". En Miguel Ángel Martín López (coord.) Los Saraguros: cosmovisión, salud e identidad andina. Una mirada desde sí mismos. Córdoba: Diputación de Córdoba, pp. 63-74.

Bidwell, Nicola J. et al. (2011) "Pleasecall ME.N.U.4EVER:Designingfor 'Callback' in Rural Africa". Proceedings of The 10 International WorkshoponInternalisation of Products and Systems. Malasya, pp. 117-138.

De Salvador Agra, Saleta y Martínez Suárez, Yolanda (2015a) "Nomadism and intermittentubiquity in 'off thegrid' Shuarpeople". Communication\&Society 28(4), pp. 87-107. https://www.unav.es/fcom/communication-society/en/articulo.php?art_id=549 [Consultado el 20 de abril de 2017].

De Salvador Agra, Saleta y Martínez Suárez, Yolanda (2015b) “Apropiaciones comunitarias en los indígenas shuar". Revista Internacional de Comunicación y Desarrollo 1(1), pp. 41-49. http://www.usc.es/revistas/index.php/ricd/article/view/2178 [Consultado el 20 de abril de 2017].

Japón Gualán, Rodrigo (2007) “¿Quién es el runa Saraguro?”. En Miguel Ángel Martín López (coord.) Los Saraguros: cosmovisión, salud e identidad andina. Una mirada desde sí mismos. Córdoba: Diputación de Córdoba, pp. 39- 50.

Lasén, Amparo (2009) “Tecnologías afectivas: de cómo los teléfonos móviles participan en la constitución de subjetividades e identidades”. En Gabriel Gatti e Iñaki Martínez de Albenil y Benjamín Tejerina (eds.) Tecnología, cultura experta e identidad en la sociedad del conocimiento. Bilbao: Universidad del País Vasco, pp. 215-248.

Martín Barbero, Jesús (1987) De los medios a las mediaciones. Comunicación, cultura y hegemonía. México: Gustavo Gili.

Martínez Suárez, Yolanda; De Salvador Agra, Saleta y De Salvador González, Xabier (2015) “Triplemente marcadas: desconexiones comunicativas en la Amazonía sur ecuatoriana”. Cuadernos.info, 36, pp. 89-107. http://www.cuadernos.info/index.php/ CDI/article/view/716/pdf [Consultado el 20 de abril de 2017]

Palacios, Ma Inés (1999) Papel de la mujer en la adaptación de colonos de la Amazonía. Quito: Abya-Yala.

Paqui Cartuche, Ángel Hipólito y Poma Guamán, María Francisca (2013) “La pérdida de la identidad cultural del pueblo kichwa Saraguro en la comunidad Cisam, parroquia Nuevo Quito, cantón Paquisha”. Trabajo de grado. Ciencias de la Educación con Mención en Educación Intercultural Bilingüe. Universidad de Cuenca. 
Provencio, Lucía (2006a) "Ecuatorianas en España y Región de Murcia. Sendas de Identidad: Diálogos de tres generaciones de mujeres quichua Saraguro". Anales de historia contemporánea 22, pp. 79-103.

Provencio, Lucía (2006b) "Ni iguales, ni idénticas. Migraciones de mujeres indígenas saraguras: De Ecuador a la Región de Murcia”. Tiempos de América 13, pp. 133-150.

Quishpe Bolaños, Marcelo (2007) "Cambios y nuevos elementos de valoración de la identidad en los Saraguro”. En Miguel Ángel Martín López (Coord.). Los Saraguros: cosmovisión, salud e identidad andina. Una mirada desde sí mismos. Córdoba: Diputación de Córdoba, pp. 53-62.

Sarango Macas, Fernando (1995) "Los Saraguro". En José Almeida Vinueza (coord.) Identidades indias en el Ecuador contemporáneo. Quito: Ediciones Abya-Yala, pp. 339369.

Smith Belote, Linda y Belote, Jim (2000) "Fuga desde abajo: Cambios individuales de identidad étnica en el sur del Ecuador". En Andrés Guerrero (comp.) Etnicidades. Quito: Flacso, pp. 81-118. 